\title{
Wave Speeds and Green's Tensors for Shear Wave Propagation in Incompressible, Hyperelastic Materials with Uniaxial Stretch
}

\author{
Ned C. Rouze, ${ }^{1}$ Annette Caenen, ${ }^{2,3}$ and Kathryn R. Nightingale ${ }^{1}$ \\ ${ }^{1}$ Biomedical Engineering, Duke University, Durham, NC 27708-0281 USA \\ ${ }^{2}$ IBiTech-bioMMeda, Ghent University, Ghent, Belgium \\ ${ }^{3}$ Biomedical Engineering, Department of Cardiology, Erasmus MC, University Medical Center Rotterdam, \\ Rotterdam, The Netherlands
}

\begin{abstract}
Assessing elastic material properties from shear wave propagation following an acoustic radiation force impulse (ARFI) excitation is difficult in anisotropic materials because of the complex relations among the propagation direction, shear wave polarizations, and material symmetries. In this paper, we describe a method to calculate shear wave signals using Green's tensor methods in an incompressible, hyperelastic material with uniaxial stretch. Phase and group velocities are determined for SH and SV propagation modes as a function of stretch by constructing the equation of motion from the Cauchy stress tensor determined from the strain energy density. The Green's tensor is expressed as the sum of contributions from the $\mathrm{SH}$ and $\mathrm{SV}$ propagation modes with the $\mathrm{SH}$ contribution determined using a closed-form expression and the SV contribution determined by numerical integration. Results are presented for a MooneyRivlin material model with a tall Gaussian excitation similar to an ARFI excitation. For an experimental configuration with a tilted material symmetry axis, results show that shear wave signals exhibit complex structures such as shear splitting that are characteristic of both the $\mathrm{SH}$ and $\mathrm{SV}$ propagation modes.
\end{abstract}

\section{INTRODUCTION}

Elastic properties of materials can be measured by observing shear wave propagation following an acoustic radiation force impulse (ARFI) excitation and relating the shear wave signals to a model of the material. This process is difficult in anisotropic materials because of the complex relations among the propagation direction, shear wave polarizations, and material symmetries. For example, compressed [1], [2] and stretched [3] phantoms exhibit transverse isotropy because the deformation introduces a symmetry axis in the material. Muscle is also an example of a transversely isotropic (TI) material with the symmetry axis determined by the direction of the muscle fibers. It can be shown that three material parameters are required to characterize an incompressible, TI material [4].

Typically, experiments in TI materials have been performed using an experimental geometry such as shown in Fig. 1(a) (see also Fig. 2 of Chatelin, et al. [3]) where SH propagation mode is observed in a plane perpendicular to the excitation axis, and the material symmetry axis is positioned in the same plane. The shear wave speeds observed in these experiments measure the group speeds for propagation along and across the elliptical shear wave propagation and are sensitive to only two of the three material parameters that characterize the material. More complicated geometries using a tilted symmetry axis as shown in Fig. 1(b) observe both the SH and SV propagation modes and are sensitive to all three material parameters that characterize an incompressible, TI material [4], [5].

In this paper, we describe a method to calculate shear wave displacements for wave propagation in an incompressible, hyperelastic material with uniaxial stretch. Previous calculations of shear wave displacement signals using Green's tensor methods have been used by Chatelin, et al. [6] based on the special-case expressions for the Green's tensor from Vavryčuk [7]. However, this special case is not sufficiently general to fully describe the shear wave signals that can be measured with the tilted symmetry axis in Fig. 1(b) [5] .

\section{BACKGROUND AND THEORY}

\section{A. Finite, uniaxial stretch}

We consider an incompressible, hyperelastic material with uniaxial stretch $\lambda$ so that a point with coordinates $(X, Y, Z)=$ $\left(X_{1}, X_{2}, X_{3}\right)$ in the reference configuration has a position $(x, y, z)=\left(x_{1}, x_{2}, x_{3}\right)$ in the stretched configuration,

$$
x=X / \sqrt{\lambda}, \quad y=Y / \sqrt{\lambda}, \text { and } z=\lambda Z .
$$

The deformation gradient tensor $\mathbf{F}$ is given by

$$
F_{i A}=\frac{\partial x_{i}}{\partial X_{A}}, \quad \mathbf{F}=\left(\begin{array}{ccc}
1 / \sqrt{\lambda} & 0 & 0 \\
0 & 1 / \sqrt{\lambda} & 0 \\
0 & 0 & \lambda
\end{array}\right) .
$$

This expression can be used to calculate the left Cauchy-Green deformation tensor $\mathbf{B}=\mathbf{F F}^{T}$ and invariants $I_{1}=\operatorname{tr} \mathbf{B}, I_{2}=$ $\frac{1}{2}\left[(\operatorname{tr} \mathbf{B})^{2}-\operatorname{tr}\left(\mathbf{B}^{2}\right)\right]$, and $I_{3}=\operatorname{det} \mathbf{B}$. For the incompressible material considered in this study, $I_{3}=1$.

\section{B. Wave motion superimposed on the uniaxial stretch}

We consider plane wave propagation at an angle $\theta$ relative to the $\widehat{z}=\widehat{A}$ axis in the $\widehat{x}-\widehat{z}$ plane, i.e., the plane shown in red in Fig. 1. Wave motion is characterized by the SH, SV, and 



Fig. 1. (a) Experimental configuration commonly used for investigations of shear wave propagation in an incompressible, TI material. The ARFI excitation force $\vec{F}$ is directed along the $Z$ axis and the material symmetry axis $\widehat{A}$ and propagation direction $\widehat{n}$ are in the $Z=0$ plane. Polarization vectors of the $\mathrm{SH}, \mathrm{SV}$, and $\mathrm{P}$ propagation modes are defined relative to the $\widehat{A}-\widehat{n}$ plane shown in red. Ultrasonic tracking measures the $Z$ component of the shear wave displacement signal and is sensitive only to the $\mathrm{SH}$ propagation mode (b) A more complicated experimental configuration in which the material symmetry axis $\widehat{A}$ is tilted relative to the $Z=0$ plane. Measurements of the $Z$ component of shear wave displacement are sensitive to both the $\mathrm{SH}$ and $\mathrm{SV}$ propagation modes.

$\mathrm{P}$ propagation modes defined by their polarization directions relative to the $\widehat{x}-\widehat{z}$ plane. For the incompressible material considered here, the $\mathrm{P}$ wave with longitudinal polarization has infinite wave speed and zero amplitude, and it will not be considered further.

Using a tilde to indicate quantities with the superimposed wave motion, the deformation $\widetilde{\mathbf{x}}$ is given by the relation

$$
\widetilde{\mathbf{x}}=\mathbf{x}+\widehat{P}^{S H} \mathcal{A}_{S H} f(\eta, t)+\widehat{P}^{S V} \mathcal{A}_{S V} g(\eta, t)
$$

where $\mathbf{x}$ is the deformation (1) without wave motion, $\widehat{P}^{S H}$ and $\widehat{P}^{S V}$ are polarization vectors, $\mathcal{A}_{S H}$ and $\mathcal{A}_{S V}$ are amplitudes, and the functions $f(\eta, t)$ and $g(\eta, t)$ are plane waves functions of the position $\eta=\widehat{n} \cdot \vec{x}$ along the propagation direction $\widehat{n}$ for the $\mathrm{SH}$ and $\mathrm{SV}$ propagation modes, respectively. The quantities $\widetilde{\mathbf{F}}, \widetilde{\mathbf{B}}, \widetilde{I}_{1}$, and $\widetilde{I}_{2}$ can be calculated from (3) using (2) and relations following (2) above.

\section{Cauchy stress tensor and the equation of motion}

Isotropic, hyperelastic materials can be characterized by a strain energy density $W$ which is a function of the invariants $I_{1}, I_{2}$, and $I_{3}$ of $\mathrm{B}$ [8]. Here, we use $I_{3}=1$ for an incompressible material so that the strain energy density for the stretched material with wave motion from section II.B can be written as $\widetilde{W}=\widetilde{W}\left(\widetilde{I}_{1}, \widetilde{I}_{2}\right)$. The Cauchy stress tensor $\widetilde{\mathbf{T}}$ can be determined from $\widetilde{W}$ by the relation [8]

$$
\widetilde{\mathbf{T}}=-p \mathbf{1}+2 \frac{\partial \widetilde{W}}{\partial \widetilde{I}_{1}} \widetilde{\mathbf{B}}-2 \frac{\partial \widetilde{W}}{\partial \widetilde{I}_{2}} \widetilde{\mathbf{B}}^{-1}
$$

where $p$ is an undetermined pressure. The equation of motion for a point at postion $\widetilde{\mathbf{x}}$ is given by the divergence of $\widetilde{\mathbf{T}}$,

$$
\rho \frac{\partial^{2} \widetilde{x}_{i}}{\partial t^{2}}=\frac{\partial \widetilde{T}_{i j}}{\partial \widetilde{x}_{j}}
$$

\section{Phase and group velocities}

Equations of motion for the $\mathrm{SH}$ and $\mathrm{SV}$ wavefunctions can be found by combining (3), (4), and (5) and assuming infinitesimal wave amplitudes. For the $\mathrm{SH}$ propagation mode,

$$
\rho \frac{\partial^{2}}{\partial t^{2}} f(\eta, t)=\rho v_{S H}^{2} \frac{\partial^{2}}{\partial \eta^{2}} f(\eta, t)
$$

where the phase velocity $v_{S H}$ is given by

$$
\begin{aligned}
\rho v_{S H}^{2}=\left(2 \lambda^{2}\right. & \left.\frac{\partial W}{\partial I_{1}}+2 \lambda \frac{\partial W}{\partial I_{2}}\right) \cos ^{2} \theta \\
& +\left(\frac{2}{\lambda} \frac{\partial W}{\partial I_{1}}+2 \lambda \frac{\partial W}{\partial I_{2}}\right) \sin ^{2} \theta .
\end{aligned}
$$

Note that the derivatives in (7) (and (9), below) are evaluated in the stretched material, without including the wave motion. Expression (7) has the same form as the $\mathrm{SH}$ phase velocity for wave propagation in an incompressible, TI material [4],

$$
\rho v_{S H}^{2}=\mu_{\|} \cos ^{2} \theta+\mu_{\perp} \sin ^{2} \theta
$$

where $\mu_{\|}$and $\mu_{\perp}$ are shear moduli that characterize wave propagation parallel and perpendicular to the symmetry axis. Thus, the group shear wave speed for the SH wave will have an elliptical shape with speeds along the semi-major and semiminor axes of $V_{\|}=\sqrt{\mu_{\|} / \rho}$ and $V_{\perp}=\sqrt{\mu_{\perp} / \rho}$, respectively.

Similarly, the SV phase velocity is given by the relation

$$
\begin{aligned}
\rho v_{\mathrm{SV}}^{2}=( & \left.2 \lambda^{2} \frac{\partial W}{\partial I_{1}}+2 \lambda \frac{\partial W}{\partial I_{2}}\right) \cos ^{2} \theta \\
+ & \left(\frac{2}{\lambda} \frac{\partial W}{\partial I_{1}}+\frac{2}{\lambda^{2}} \frac{\partial W}{\partial I_{2}}\right) \sin ^{2} \theta \\
+ & 4\left[\left(\lambda^{2}-\frac{1}{\lambda}\right)^{2} \frac{\partial^{2} W}{\partial I_{1}^{2}}+\left(\lambda-\frac{1}{\lambda^{2}}\right)^{2} \frac{\partial^{2} W}{\partial I_{2}^{2}}\right. \\
+ & \left.2\left(\lambda^{2}-\frac{1}{\lambda}\right)\left(\lambda-\frac{1}{\lambda^{2}}\right) \frac{\partial^{2} W}{\partial I_{1} \partial I_{2}}\right] \sin ^{2} \theta \cos ^{2} \theta
\end{aligned}
$$

\section{E. Calculation of the Green's tensor}

Neglecting the P propagation mode, components $G_{i n}(\vec{r}, \omega)$ of the Green's tensor in the temporal frequency domain can be written a sum of the SH and SV propagation modes as [5]

$$
\begin{aligned}
G_{i n}(\vec{r}, \omega) & =G_{i n}^{S H}(\vec{r}, \omega)+G_{i n}^{S V}(\vec{r}, \omega) \\
& =\sum_{N=S H, S V} \frac{1}{8 \pi^{3}} \iiint \frac{\widehat{P}_{i}^{N} \widehat{P}_{n}^{N}}{\rho k^{2} v_{N}^{2}-\rho \omega^{2}} e^{i \vec{k} \cdot \vec{r}} d^{3} k
\end{aligned}
$$

where $\widehat{P}^{S H}$ and $\widehat{P}^{S V}$ are polarization vectors, and the phase velocities $v_{S H}$ and $v_{S V}$ are given by (7) and (9), respectively.

For the SH propagation mode, it can be shown [5], [9] that $G_{i n}^{S H}(\vec{r}, \omega)$ is given by the closed-form expression

$$
\begin{aligned}
G_{i n}^{S H}(\vec{r}, \omega)= & \frac{1}{4 \pi \mu_{\perp}}\left(\delta_{i n}-\frac{r_{i} r_{n}}{r_{\perp}^{2}}\right) \frac{e^{-i k_{0} r^{\prime}}}{r^{\prime}} \\
& -\frac{i}{4 \pi \mu_{\|}}\left(\delta_{i n}-\frac{2 r_{i} r_{n}}{r_{\perp}^{2}}\right) \frac{e^{-i k_{0} r^{\prime}}-e^{-i k_{0}|z|}}{k_{0} r_{\perp}^{2}}
\end{aligned}
$$


where $r_{\perp}^{2}=x^{2}+y^{2}, r^{\prime 2}=r_{\perp}^{2} \mu_{\perp} / \mu_{\|}+z^{2}, k_{0}^{2}=\rho \omega^{2} / \mu_{L}$, and the sign of $k_{0}$ has been chosen to give outgoing waves when combined with the $e^{+i \omega t}$ time dependence.

We have not found a closed-form expression for $G^{S V}(\vec{r}, \omega)$. Instead, it can be shown [5] that $G_{i n}^{S V}(\vec{r}, \omega)$ are given by

$$
\begin{aligned}
G_{i n}^{S V}(\vec{r}, \omega)= & \frac{-i \omega}{16 \pi^{2} \rho v_{0}^{3}} I_{i n}^{S}\left(\alpha, \theta_{r}, \Delta_{S V}\right) \\
& +\frac{1}{8 \pi^{2} \rho r v_{0}^{2}} I_{i n}^{L}\left(\theta_{r}, \Delta_{S V}\right)
\end{aligned}
$$

where $v_{0}=\sqrt{\mu_{\|} / \rho}$ is the phase velocity along the symmetry axis, $\alpha=r \omega / v_{0}, \Delta_{S V}$ is a function of the material parameters and angle $\theta_{k}$ determined from (9), and the surface and line integrals are given by

$$
\begin{aligned}
& I_{i n}^{S}\left(\alpha, \theta_{r}, \Delta_{S V}\right) \\
& =\int_{0}^{2 \pi} \int_{0}^{\pi} \frac{\widehat{P}_{i}^{S V} \widehat{P}_{n}^{S V}}{\left(1+\Delta_{S V}\right)^{3 / 2}} e^{-i \alpha|\widehat{k} \cdot \widehat{r}| / \sqrt{1+\Delta_{S V}}} \sin \theta_{k} d \theta_{k} d \phi_{k}
\end{aligned}
$$

and

$$
I_{i n}^{L}\left(\theta_{r}, \Delta_{S V}\right)=\int_{0}^{2 \pi} \int_{0}^{\pi} \frac{\widehat{P}_{i}^{S V} \widehat{P}_{n}^{S V}}{1+\Delta_{S V}} \delta(\widehat{k} \cdot \widehat{r}) \sin \theta_{k} d \theta_{k} d \phi_{k} .
$$

\section{Methods}

\section{A. Mooney-Rivlin material}

In this paper, we apply the methods described in Section II to the case of a Mooney-Rivlin material model with strain energy density given by the relation

$$
W=C_{10}\left(I_{1}-3\right)+C_{01}\left(I_{2}-3\right) .
$$

Specifically, we present results using the material parameters $C_{10}=5.0 \mathrm{kPa}$ and $C_{01}=-1.0 \mathrm{kPa}$. We note that for this material model, only first order derivatives of $W$ with respect to $I_{1}$ and $I_{2}$ are nonzero in (9), and the phase velocities for the SV propagation modes have the same form as for the SH propagation mode, though the values of $\mu_{\|}$and $\mu_{\perp}$ in (8) are different. Thus, the group shear wave speeds for the SV propagation will be elliptical as for the SH mode.

\section{B. Calculation of the shear wave displacement signals}

The shear wave signal $\vec{u}\left(\vec{r}_{o b s}, t\right)$ at an observation position $\vec{r}_{o b s}$ and time $t$ was calculated by dividing the excitation force $\vec{f}\left(\vec{r}_{s}, t_{s}\right)$ into spatial and temporal source voxels $s$ and summing the contributions from each voxel. The Greens tensor $G_{i n}\left(\vec{r}_{o b s}-\vec{r}_{s}, t-t_{s}\right)$ gives the relative contribution to the signal from each source voxel. Assuming the force can be factored into a spatial function $\vec{f}\left(\vec{r}_{s}\right)$ and temporal window $W(t)$, components $u_{i}\left(\vec{r}_{o b s}, \omega\right)$ of the shear wave signal can be written in the temporal frequency domain as

$$
u_{i}\left(\vec{r}_{o b s}, \omega\right)=\sum_{s} G_{i n}(\vec{r}, \omega) f_{n}\left(\vec{r}_{s}\right) W(\omega)
$$

where $\vec{r}=\vec{r}_{o b s}-\vec{r}_{s}$ is the relative position between the source and observation positions and summation over the component index $n$ of the force is implied.
To simplify these calculations, we distinguish between two coordinate systems; the $(x, y, z)=\left(x_{1}, x_{2}, x_{3}\right)$ coordinate system defined by the material symmetry axis $\widehat{A}$ and propagation direction $\widehat{n}$ used to calculate the Green's tensor in (10), and a second $(X, Y, Z)=\left(X_{1}, X_{2}, X_{3}\right)$ coordinate system to describe the experimental configuration as in Fig. 1. Defining a rotation matrix $R$ with elements $R_{A i}=\widehat{X}_{A} \cdot \widehat{x}_{i}$ to transform from the $x y z$ to $X Y Z$ coordinate system gives [5]

$$
\begin{aligned}
& u_{A}\left(\vec{r}_{o b s}, \omega\right)=\sum_{s} R_{A i} {\left[G_{i n}^{S H}(\vec{r}, \omega)+G_{i n}^{S V}(\vec{r}, \omega)\right] } \\
& \times\left(R^{-1}\right)_{n B} f_{B}\left(\vec{r}_{s}\right) W(\omega) .
\end{aligned}
$$

In practice, the surface (13) and line (14) integral functions were precomputed. Then, when performing the sum over source positions in (17), values of $I_{i n}^{S}\left(\alpha, \theta_{r}, \Delta_{S V}\right)$ and $I_{i n}^{L}\left(\theta_{r}, \Delta_{S V}\right)$ were evaluated by interpolation with an efficiency comparable to the evaluation of a closed-form expression. In addition, because the surface and line integral functions are parameterized, the evaluation of these functions is reduced by a factor of roughly $10^{6}$ compared to the calculation of the integrals for each combination of $r_{s}, r_{o b s}$, and $\omega$ in (17).

\section{Ultrasonic excitation and tracking}

Results are presented in Section IV for the case of a tall Gaussian excitation directed in the $\widehat{Z}$ direction as in Fig. 1,

$$
\vec{f}\left(\vec{r}_{s}, t\right)=e^{-X^{2} / 2 \sigma_{X}^{2}} e^{-Y^{2} / 2 \sigma_{Y}^{2}} e^{-Z^{2} / 2 \sigma_{Z}^{2}} W(t) \widehat{Z}
$$

where $\sigma_{X}=\sigma_{Y}=0.4 \mathrm{~mm}$ and $\sigma_{Z}=7.5 \mathrm{~mm}$. A $200 \mu \mathrm{s}$ rectangular excitation window $W(t)$ was used. Results show only the $Z$-component of the shear wave displacement signal as would be observed with ultrasonic tracking.

\section{RESULTS AND DISCUSSION}

Figure 2 shows the phase velocities (left) for the SH and SV propagation modes calculated using (7) and (9), respectively, for the Mooney-Rivlin described in Section III-A with stretch $\lambda=1.8$. The group velocities (right) are calculated from the phase velocities using the standard procedure [4]. As noted in Section III.A, both the SH and SV group velocities are elliptical because second order derivatives in (9) are zero for the Mooney-Rivlin material (15).
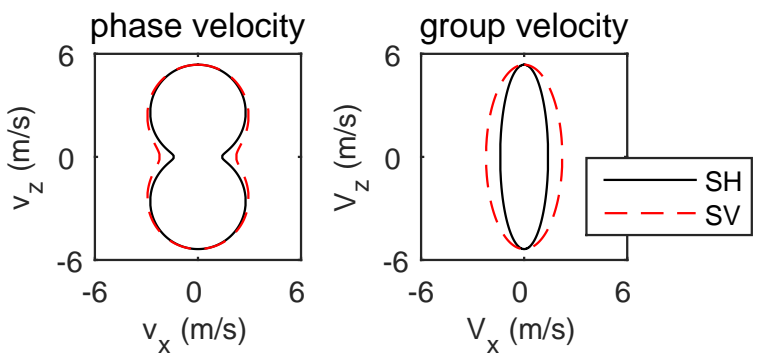

Fig. 2. Phase (left) and group (right) velocities for the SH and SV progagation modes in the $\widehat{A}-\widehat{n}$ (red) plane in figure 1 for propagation in a Mooney-Rivlin material (15) with $C_{10}=5.0 \mathrm{kPa}, C_{01}=-1.0 \mathrm{kPa}$, and $\lambda=1.8$. 


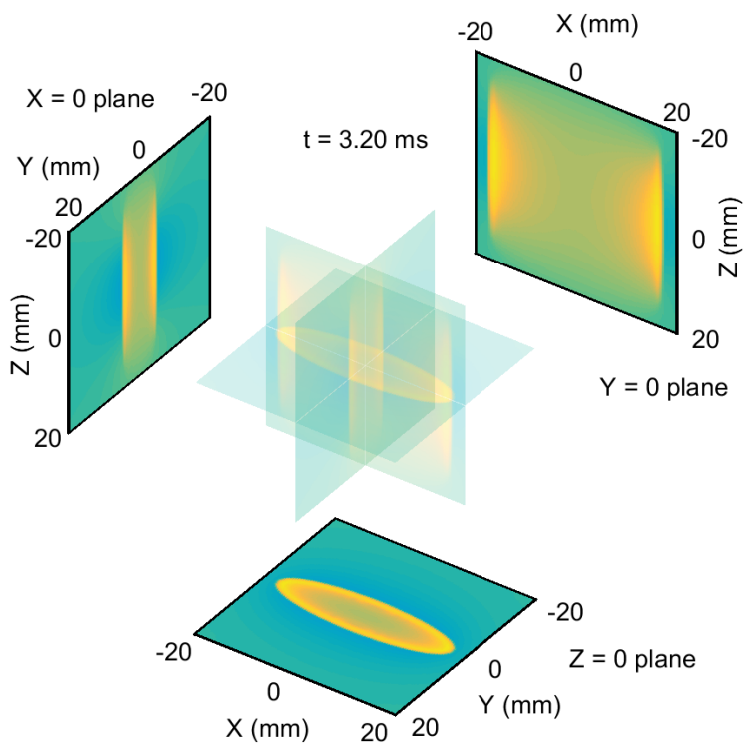

Fig. 3. Views of shear wave signals in the $X=0, Y=0$, and $Z=0$ planes at time $t=3.20 \mathrm{~ms}$ from the tall Gaussian excitation. The planes overlap and are shown with semi-transparency in the center, and are also duplicated without transparency at positions displaced from the center. The experimental configuration is shown in Fig. 1(a) with the material symmetry axis positioned along the $X$ axis and the excitation force directed along the $\widehat{Z}$ direction. The $\widehat{Z}$ component of displacement is shown.

Figure 3 shows a snapshot of the shear wave displacement signal in the $X=0, Y=0$, and $Z=0$ planes from the tall Gaussian excitation at a time $t=3.20 \mathrm{~ms}$. Signals are shown as overlapping, semi-transparent planes at the center, and are duplicated without transparency at positions displaced from the center. The experimental configuration is shown in Fig. 1(a) with the material symmetry axis positioned along the $X$ axis and the excitation force directed along the $\widehat{Z}$ direction. The $\widehat{Z}$ component of displacement is shown. Only the $\mathrm{SH}$ propagation mode contributes to the signals in the $Z=0$ plane, thereby giving the expected elliptical shape.

Figure 4 shows shear wave signals observed with the experimental setup shown in Fig. 1(b) where the material symmetry axis is tilted at an angle of $45^{\circ}$ with respect to the $Z=0$ plane. As in Fig. 3, only the $\widehat{Z}$ component of displacement is shown. However, in this case, both the SH and SV propagation modes contribute to the shear wave signals observed in the $Z=0$ plane and give shear splitting for propagation along the $Y$-axis

\section{CONCLUSION}

This study describes the calculation of phase velocities and shear wave signals following an ARFI excitation in an incompressible, hyperelastic material with uniaxial stretch. By using an experimental configuration with the material symmetry axis tilted relative to the exciation axis, both the $\mathrm{SH}$ and SV propagation modes can be observed, thereby providing additional information regarding the material properties of stretched phantoms, and of anisotropic tissue such as skeletal muscle.

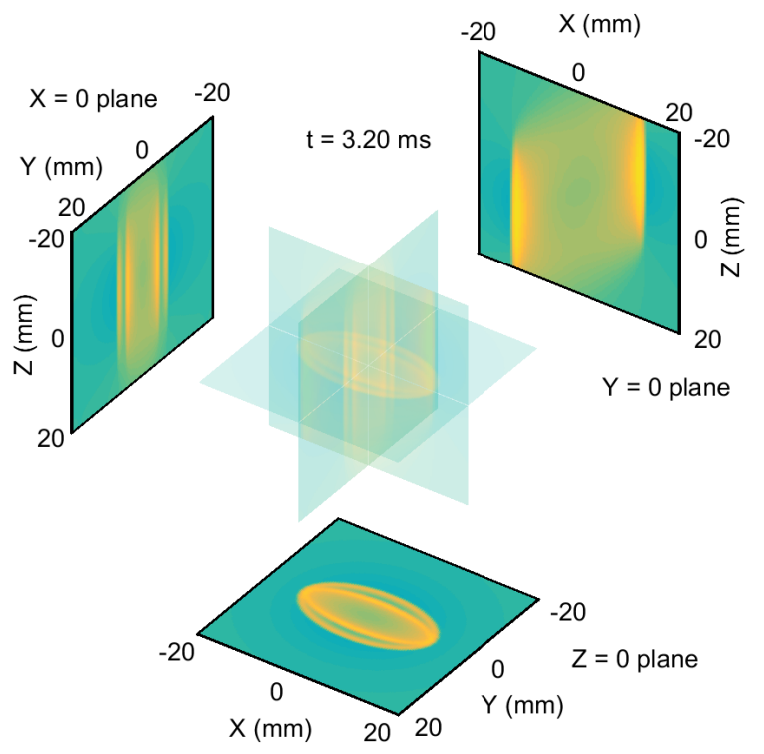

Fig. 4. Shear wave signals for the same case as in Fig. 3 with the excitation setup shown in Fig. 1(b).

\section{ACKNOWLEDGMENTS}

The authors thank Derek Chan, Courtney Trutna, Mark Palmeri, and Fabrice Prieur for helpful discussions. This work was supported in part by National Institutes of Health grants R01-EB022106 and R01-CA142824, and by grants from the Flemish Government Agency for Innovation and Entrepreneurship (VLAIO) and Research Foundation Flanders (FWO).

\section{REFERENCES}

[1] J.-L. Gennisson, M. Rénier, S. Catheline, C. Barrière, J. Bercoff, M Tanter and M. Fink, Acoustoelasticity in Soft Solids: Assessment of the Nonlinear Shear Modulus with the Acoustic Radiation Force, J. Acoust. Soc. Am. vol. 122, pp. 3211-3219, 2007.

[2] M. W. Urban, M. Lopera, S. Aristizabal, C. Amador, I. Nenadic, R. R. Kinnick, A. D. Weston, B. Qiang, X. Zhang, and J. F. Greenleaf, "Characterization of transverse isotropy in compressed tissue-mimicking phantoms," IEEE Trans. Ultrason. Feroelectr. Freq. Control vol. 62, pp. 1036-1046, 2015.

[3] S. Chatelin, M. Bernal M, T. Deffieux, C. Papadacci, P. Flaud, A. Nahas, C. Boccara, J.-L. Gennisson, M. Tanter, and M. Pernot, "Anisotropic polyvinyl alcohol hydrogel phantom for shear wave elastography in fibrous biological soft tissue: a multimodality characterization," Phys. Med. Biol. vol. 59 pp. 6923-6940, 2014.

[4] N. C. Rouze, M. H. Wang, M. L. Palmeri, and K. R. Nightingale, "Finite element modeling of impulsive excitation and shear wave propagation in an incompressible, transversely isotropic medium,” J. Biomech. vol. 46, pp. 2761-2768, 2013.

[5] N. C. Rouze, M. L. Palmeri, and K. R. Nightingale, "Tractable calculation of the Green's tensor for shear wave propagation in an incompressible, transversely isotropic material," submitted to Phys. Med. Bio.

[6] S. Chatelin, J.-L. Gennisson, M. Bernal, M. Tanter, and M. Pernot, "Modelling the impulse diffraction field of shear waves in transverse isotropic viscoelastic medium," Phys. Med. Biol. vol. 60, pp. 3639-3654, 2015.

[7] V. Vavryčuk, "Asymptotic Green's function in homogeneous anisotropic viscoelatic media.” Proc. R. Soc. A vol. 463, pp. 2689-2707, 2007.

[8] G. A. Holzapfel, Nonlinear Solid Mechanics, NY: Wiley, 2000, Chap. 6.

[9] D. Gridin, "Far-field asymptotics of the Green's tensor for a transversely isotropic solid," Proc. R. Soc. Lond. A vol. 456, pp. 571-591, 2000. 